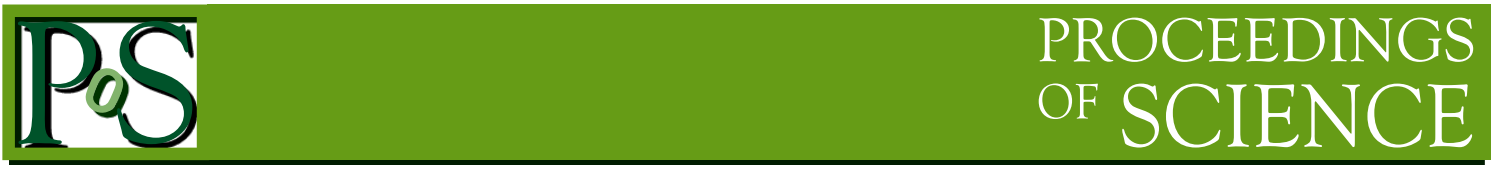

\title{
International Linear Collider (ILC)
}

- Technical Progress and Prospect -

\author{
Akira Yamamoto for the LCC Collaboration ${ }^{1}$ \\ High Energy Accelerator Research Organization (KEK) \\ 1-1 Oho, Tsukuba, Ibaraki, Japan \\ E-mail:akira.yamamoto@kek.jp
}

Technical progress in the ILC accelerator design and R\&D efforts are overviewed and superconducting $\mathrm{RF}$ and nano-beam technologies are. focused as core technologies. Future prospects are briefly discussed.

38th International Conference on High Energy Physics 3-10 August 2016

Chicago, USA

\section{${ }^{1}$ Akira Yamamoto}




\section{Introduction}

International Linear Collider (ILC) is an energy-frontier electron-positron collider based on two key technologies of superconducting radio-frequency (SRF) and nano-beam technologies. The center-of-mass energy (C.E.) is expected in a range of $200-500 \mathrm{GeV}$, extendable to $1 \mathrm{TeV}$ $[1,2]$. The total length of the accelerator complex is $31 \mathrm{~km}$ in the C.E. $500 \mathrm{GeV}$ baseline design and $50 \mathrm{~km}$ in the $1 \mathrm{TeV}$ energy upgrade. It will provide energy frontier particle physics experiments with very clean environment, and will enable very precise study of Higgs particles and physics beyond the standard model.

The accelerator system is composed of i) a polarized electron and positron sources ii) damping rings (DR) at $5 \mathrm{GeV}$, iii) beam transport to the main linacs followed by a two-stage bunch-compressor system accelerating the beam up to $15 \mathrm{GeV}$, iv) two $11 \mathrm{~km}$ main linacs accelerating the beam up to $250 \mathrm{GeV} / 500 \mathrm{GeV}$, utilizing $1.3 \mathrm{GHz}$ SRF cavities operating at an average gradient of $31.5 \mathrm{MV} / \mathrm{m}$, and $\mathrm{v}$ ) beam-delivery systems, each $2.2 \mathrm{~km}$ long, which bring the beams into collision with a $14 \mathrm{mrad}$ crossing angle, at a single interaction point. It will be shared with two detectors, in "push-pull" configuration. A general layout of the ILC accelerator complex with main parameters are shown in Fig. 1. These design parameters have been demonstrated during the Technical Design Phase and in past three years.

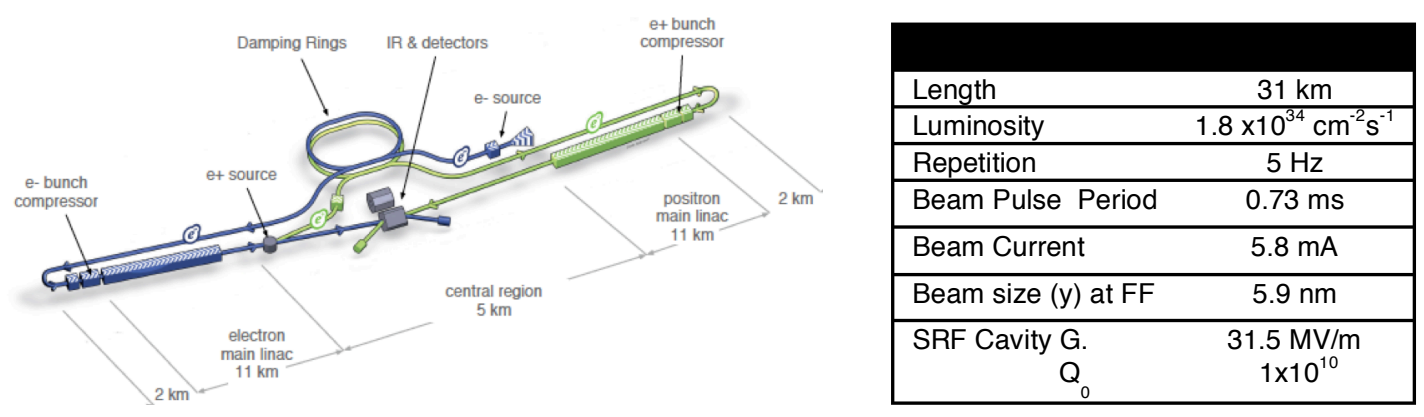

Fig. 3. ILC general layout and main parameters.

\section{SRF Technology for the ILC}

The ILC accelerator design has been optimized with the SRF technology with the following advantages [2]:

- $\quad$ Small RF surface resistance and large quality factor, Q, allowing a long pulse-duration of 1 ms with a higher duty operational factor in beam acceleration.

- Lower RF operational frequency of $1.3 \mathrm{GHz}$, leading to a larger beam-aperture of $\sim 70 \mathrm{~mm}$ in diameter and larger acceptance for high intensity beam resulting in high-luminosity.

The shape of a superconducting cavity is optimized for properties such as: (i) reduced excitation of higher-order harmonics by the beam; (ii) lower surface magnetic field to maximize the critical limit of the superconducting to normal-conducting phase transition; (iii) lower surface electric field to suppress field emission; and (iv) smaller multipacting behavior. The large iris opening and elliptical shape result from optimization of these considerations, as the crosssection. The ILC cavity design has been based on much experience from the TESLA and European XFEL programs as shown in Fig. 2 [3]. 
The ILC main linacs accelerate the beam from $15 \mathrm{GeV}$ (after pre-acceleration in bunch compressors) to a maximum energy of $250 \mathrm{GeV}$. Beam acceleration in each linac is provided by approximately 7,400 superconducting ninecell niobium cavities with a unit length of $1.27 \mathrm{~m}$ operating at $2 \mathrm{~K}$, assembled into 850 cryomodules. The average design accelerating gradient of the cavities is $31.5 \mathrm{MV} / \mathrm{m}$, for $250 \mathrm{GeV}$ operation in each linac, with a corresponding quality factor $\mathrm{Q}_{0}$ of $10^{10}$. A random cavity-to-cavity gradient spread of $+/-20 \%$ is tolerated to accommodate expected mass-production variations.

The extensive worldwide production experience both in the labs and in industry now gives confidence that these

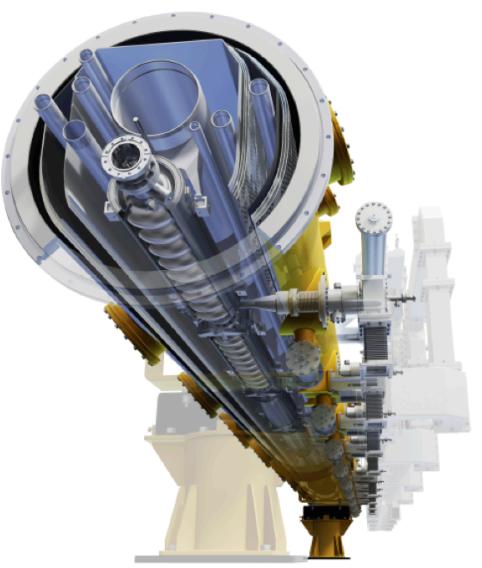

Fig. 2.SRF cavity string assembly in a cryomodule. requirements can be achieved. For an average of $31.5 \mathrm{MV} / \mathrm{m}$ operation with the nominal beam current of $5.8 \mathrm{~mA}$, the optimal matched loaded-Q $\left(\mathrm{Q}_{\mathrm{L}}\right)$ is $5.4 \times 10^{6}$. This corresponds to a cavity fill-time of $0.925 \mathrm{~ms}$, added to the nominal beam pulse width of $0.727 \mathrm{~ms}$, gives a total RF pulse length of $1.65 \mathrm{~ms}$ in the baseline design. The cavity package includes the cavity mechanical tuner integrated into the titanium helium vessel of the cavity, and an adjustable high-power coupler. In addition to a slow mechanical tuner (used for initial tuning after cool-down and slow drift compensation), a fast piezo-driven tuner is provided to dynamically adjust the frequency variation due to the cavity deformation, caused by the RF pulse, known as "Lorentz-force detuning".

The RF power is provided by $10 \mathrm{MW}$ multi-beam klystrons (MBK). A string of 39 cavities in 4.5 cryomodule is powered by a $10 \mathrm{MW}$ klystron, as shown in Fig. 3.
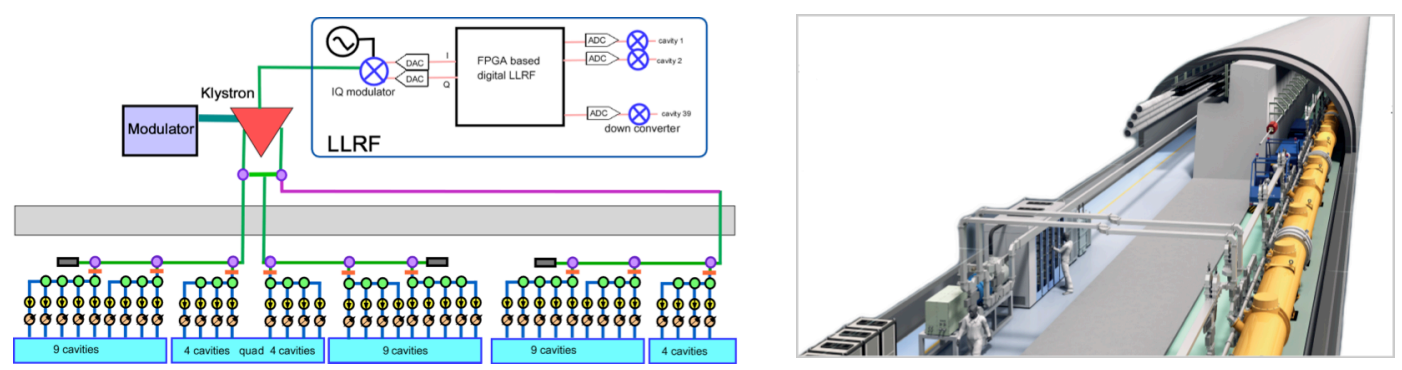

Fig. 3. A string of 39 cacities in 4.5 cryomodule powerd by a $10 \mathrm{MW}$ klystron, and the RF power transferred and distribeted by using wave guides.

Cooling of the SRF main linac is provided with10 large cryogenic plants, each of which has an equivalent cooling power of $\sim 20 \mathrm{~kW}$ at $4.5 \mathrm{~K}$. The plants are located in pairs separated by $5 \mathrm{~km}$ along the linacs, with each plant cooling $2.5 \mathrm{~km}$ of continuous linac. To simplify the liquid-helium transport, the main linacs follow the Earth's average curvature For the cavity operation at $2 \mathrm{~K}$, the SRF cavities are immersed in a saturated He-II bath. Shields cooled with helium gas intercept thermal radiation and provide a heat sink for conduction at $5-8 \mathrm{~K}$ and at $40-80 \mathrm{~K}$. Each cryomodule has an estimated $2 \mathrm{~K}$ static cryogenic heat load of $1.3 \mathrm{~W}$ while the $2 \mathrm{~K}$ dynamic heat load is approximately $9.8 \mathrm{~W}$.

Over the last 30 years, significant progress has been made and the cavity gradient performance has been significantly improved. Figure 4 (a) shows the gradient improvement with both single-cell and multi-cell cavities [4]. Various efforts of mechanical assembly and surface treatment contributed to this significant progress, as well as important efforts for the invention and 
deployment of tools to identify and repair quench-causing effects [5]. These processes have been needed to achieve both the high-gradient goal and to demonstrate a production yield of $90 \%$ worldwide to be ready for a large-scale manufacturing required for ILC. This goal has been met in the ILC technical design phase: a yield of $94 \%$ for cavity production above 28 $\mathrm{MV} / \mathrm{m}$ (as a lower threshold for $35 \mathrm{MV} / \mathrm{m}+/-20 \%$ ) and an average gradient of $37.1 \mathrm{MV} / \mathrm{m}$ has been achieved as shown in Fig. 4 (b). The yield thus corresponds to $94 \%$ for a cavity ensemble with an average gradient above $35 \mathrm{MV} / \mathrm{m}$ and complies with the allowable gradient-spread specification of $\pm 20 \%$. The yield for cavities with gradient above is $35 \mathrm{MV} / \mathrm{m}$ is $75 \%$ [1].
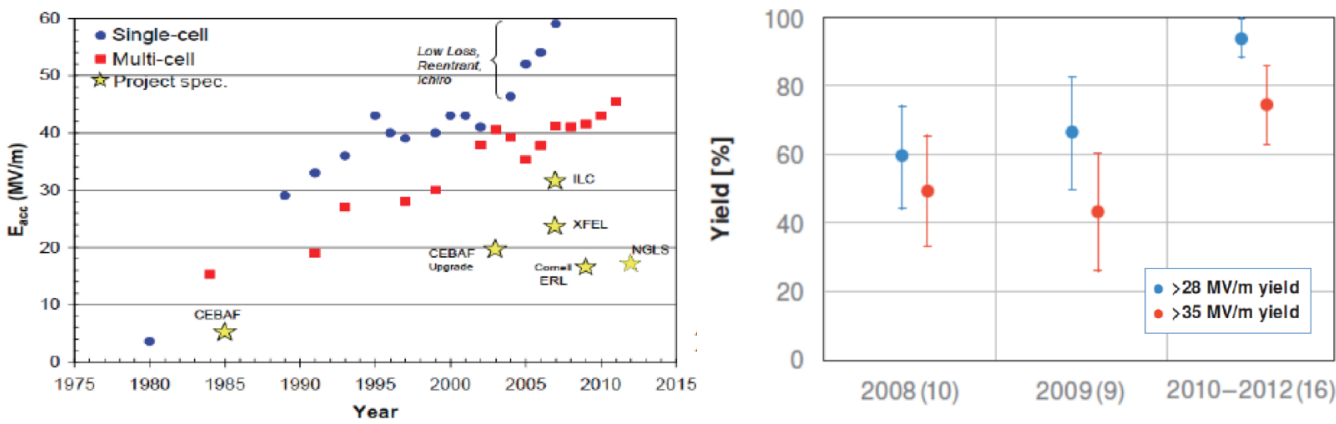

Fig. 4. (a) SRF L-band cavity-gradient progress and requirements from various projects, and (b) progress in the gradient yield at $\mathrm{G}>28 \mathrm{MV} / \mathrm{m}$ and $>35 \mathrm{MV} / \mathrm{m}$ during a period during the GDE phase in $2007-2012$.

Larger statistics on the yield has been obtained from the European XFEL cavity production program. A half of 800 cavities fabricated by Research Instruments had been surface-processed with the same recipe as that of the ILC SRF cavities, and the results may well represent the state of the technology as shown in Fig. 5. The gradient achievements through the surface treatment process are summarized in Table $\mathrm{x}$ in comparison with the ILC requirements $[6,7]$.

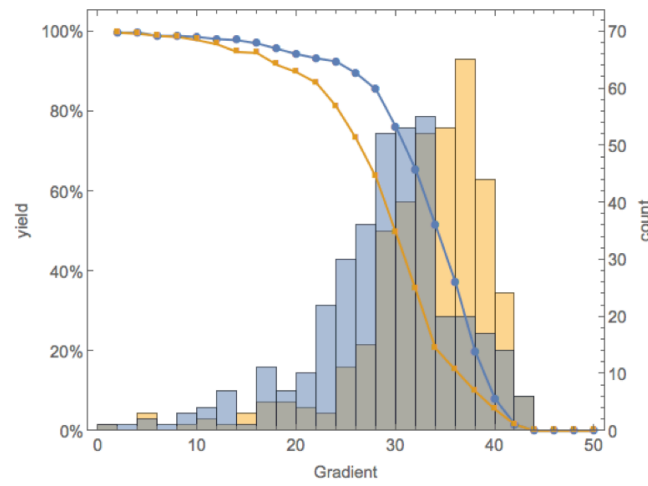

\begin{tabular}{|c|c|c|c|c|}
\hline $\begin{array}{c}\text { Process } \\
\& \\
\text { Test }\end{array}$ & $\begin{array}{c}\text { Gradient (G) } \\
\text { Yield }(\mathrm{Y})\end{array}$ & E-XFEL & $\begin{array}{c}\text { E-XFEL } \\
\left(\mathrm{Q}_{0} \geq 10^{10}\right)\end{array}$ & $\begin{array}{c}\text { ILC } \\
\text { spec. }\end{array}$ \\
\hline $1^{\text {st }}$ pass & $\begin{array}{c}\mathrm{G}_{\mathrm{av}}(\mathrm{MV} / \mathrm{m}) \\
\frac{\mathrm{Y} 28 \mathrm{MV} / \mathrm{m}}{(\%)}\end{array}$ & $\begin{array}{c}35.2 \\
\underline{85}\end{array}$ & $\begin{array}{c}33.5 \\
\underline{63}\end{array}$ & $\begin{array}{c}\geq 35 \\
\underline{75}\end{array}$ \\
\hline $1^{\text {st }}+2^{\text {nd }}$ & $\begin{array}{c}\mathrm{G}_{\mathrm{av}}(\mathrm{MV} / \mathrm{m}) \\
\frac{\mathrm{Y} 28 \mathrm{MV} / \mathrm{m}}{(\%)}\end{array}$ & $\begin{array}{c}35.0 \\
\underline{94}\end{array}$ & $\begin{array}{c}33.4 \\
\underline{82}\end{array}$ & $\begin{array}{c}\geq 35 \\
\underline{90}\end{array}$ \\
\hline $1^{\text {st }}+2^{\text {nd }}$ \\
$+3^{\text {rd }}$ & $\begin{array}{c}\mathrm{G}_{\mathrm{av}}(\mathrm{MV} / \mathrm{m}) \\
\frac{\mathrm{Y} 28 \mathrm{MV} / \mathrm{m}}{(\%)}\end{array}$ & & 33.4 & \\
\hline
\end{tabular}

Fig. 5. Gradient achieved in European XFEL in comparisons with the ILC requirements.

It should be also noted that the progress in the averaged field gradient after the 8-cavitystring assembly into the cryomodule. The gradient degradation happening in the early stage of the assembly has been successfully mitigated, after much effort given for very careful assembly procedure was established, with keeping the cleanest working environment [8].

SRF Beam test facilities have been required for major technical demonstrations with real beam acceleration. The primary goals are: the demonstration of ILC linac performance and evaluation of realistic cavity performance with beam acceleration. The Free Electron Laser 
in Hamburg (FLASH) as a soft X-ray free-electron laser facility [9] was used to demonstrate the SRF beam acceleration condition required at ILC, and the so called "9 $\mathrm{mA}$ program" has successfully demonstrated the beam acceleration parameters required for the ILC [2, 10].

The superconducting linac facility at Fermilab has demonstrated an averaged field gradient above $31.5 \mathrm{MV} / \mathrm{m}$, in the $2^{\text {nd }}$ cryomodule assembly consisting of eight 9-cell cavities [11]. S1-Global program hosted at KEK has demonstrated 8 SRF cavities, fabricated in different regions and assembled to a string in a cryomodule, and tested [12]. KEK is in preparation to demonstrate the SRF cryomodule system for the beam acceleration [13]. The ILC SRF accelerator technology has been sufficiently progressed with worldwide efforts and prepared for the ILC project realization.

\section{Nano-beam technology for the ILC}

The challenge of colliding nanometer-sized beams at the beam interaction are three distinct issues of i) creating small emittance beams, ii) preserving the emittance during beam acceleration and transport, and iii) focusing the beams to nanometers for colliding them. The Accelerator Test Facility (ATF) hosted at KEK is providing a prototype accelerator complex consisting of an electron linac, a damping ring, a beam extraction system, and the final focus beam transport line ATF2, as the layout shown in Fig. 6 [14].

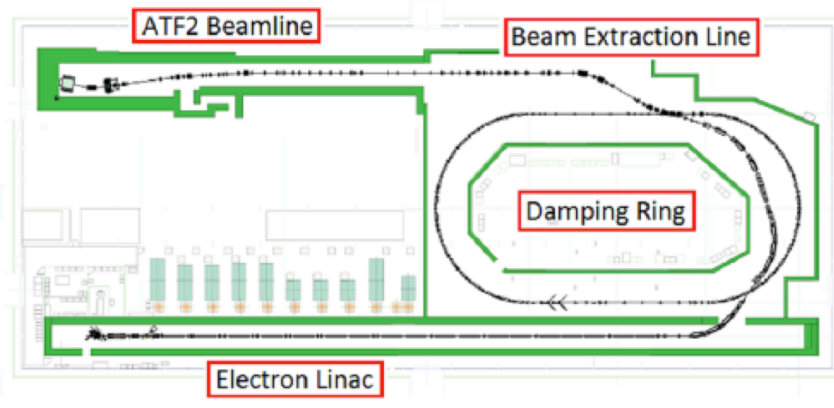

\begin{tabular}{|c|c|c|c|}
\hline \multicolumn{2}{|c|}{ ILC Parameters } & ILC & ATF2 \\
\hline Beam Energy & {$[\mathrm{GeV}]$} & 250 & 1.3 \\
\hline $\mathrm{L}^{*}$ (FFQ-IP dist.) & [m] & 4 & 1 \\
\hline V. $\beta$ function at IP & {$[\mathrm{mm}]$} & 0.48 & 0.1 \\
\hline V. emittance & [pm] & 0.07 & 0.1 \\
\hline $\begin{array}{l}\text { V. beam size at IF } \\
\text { (achieved) }\end{array}$ & {$[\mathrm{nm}]$} & 5.9 & $\begin{array}{c}37 \\
(41)\end{array}$ \\
\hline
\end{tabular}

Fig 6. ATF accelerator layout and main parameters ompared with the ILC parameters.

The damping ring is to deal with the first issue (i) and has succeeded to reach the low emittance satisfying the ILC requirements [15]. The ATF program has been extended to demonstrate the third issue (iii) to study the final focusing of the beam to nanometers. A primary goal is to establish the ILC final focus method with the same optics and with comparable beam-line tolerances, and to reach a final-focus beam size of $37 \mathrm{~nm}$ at an ATF2 beam energy of $1.3 \mathrm{GeV}$, corresponding to $5.9 \mathrm{~nm}$ at the ILC beam energy of $250 \mathrm{GeV}$. ATF2

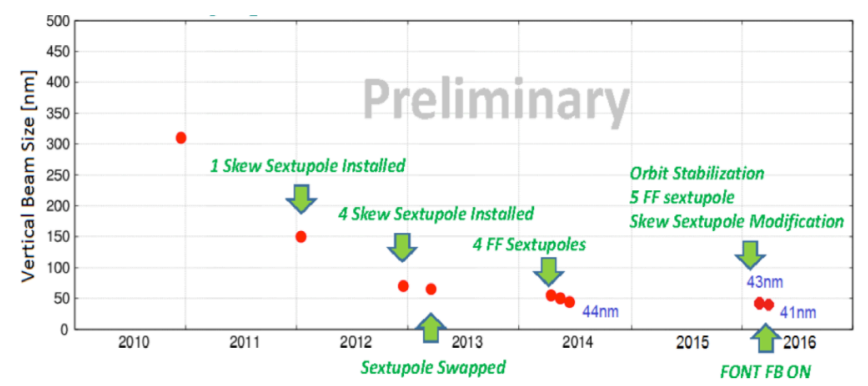

Fig. 7. Progress in the final-focus beam size at ATFachieved a vertical beam size of $55 \mathrm{~nm}$ in 2013, and achieved $41 \mathrm{~nm}$, in 2016, nearly approaching to the primary goal as shown in Fig. 7 [16-19]. 
A next important goal is in progress to develop the position stabilization at the beam collision, with a few nanometer ranges, by using bunch train feedback scheme. It should be noted that measuring transverse beam sizes of tens of nanometers at the IP requires specialized beam instrumentation, in particular a beam-size monitor, and it has been realized by using laser interferometry technology (IPBSM, referred to as a Shintake monitor [20]).

\section{Summary and Prospect}

The ILC is based on two key technologies of superconducting RF and nano-beam technologies, well integrated in over 20 years of global collaboration, as described in the ILC Technical Design Report published by the ILC Global Design Effort in 2013. Superconducting RF technology has been much matured through indusrializaiton efforts for the European XFEL accelerator construction. The nano-beam technology required has been advansed with the global ATF collaboration, and the final focus beam size have been nearly demonstrated to reach the R\&D goal. These progress are summarized in Table 1.

Table 1. Technical parameters demonstrated to prepare for the ILC realization.

\begin{tabular}{|l|c|c|l|}
\hline Characteristics & Parameter & Unit & Demonstrated woth global effort \\
\hline SRF & & & \\
\hline Av. accelerating gradient in CM & $31.5( \pm 20 \%)$ & $\mathrm{MV} / \mathrm{m}$ & DESY, FNAL, \\
\hline Cavity Q & 10 & & DESY, FNAL, \\
\hline Cavity qualification gradient & $35( \pm 20 \%)$ & $\mathrm{MV} / \mathrm{m}$ & $\mathrm{DESY}, \mathrm{FNAL}, \mathrm{JLab}$, Cornell, KEK, \\
\hline Beam current & 5.8 & $\mathrm{~mA}$ & $\mathrm{DESY}, \mathrm{KEK}$ \\
\hline Number of bunches per pulse & 1312 & & DESY \\
\hline Beam pulse length & 730 & $\mathrm{~ms}$ & DESY, KEK \\
\hline RF pulse length (incl. fill time) & 1.65 & $\mathrm{~ms}$ & DESY, KEK, FNAL \\
\hline Pulse repetition rate & 5 & $\mathrm{~Hz}$ & DESY, KEK \\
\hline Nano-beam & & & \\
\hline ILC-FF beam size (y) & $\begin{array}{c}5.9 \\
\text { KEK-ATF-FF equiv. beam size (y) }\end{array}$ & $\mathrm{nm}$ & ATF collaboration \\
\hline
\end{tabular}

In future prospect to prepare for the ILC realization, the SRF technology shall be matured in cost effective fabrication and mass-production technology. A break-through technology for surface treatment, so-called "nitrogen infusion" developed at Fermilab [21] should be further demonstrated with sufficient statics, and it may contribute to high-Q and high-G performance resulting in the cost effective cavity fabrication. For nano-beam technology, beam position stabilization for final focusing will be further R\&D goal. Further engineering design and R\&D works for positron source and beam dump are to be carried out in the preparation phase for the ILC [22].

\section{References}

[1] ILC Technical Design Report (2013): URL available: http://www.linearcollider.org/ILC/Publications/Technical-Design-Report

[2] A. Yamamoto and K. Yokoya: in "Review of Accelerator Science and Technology, eds. A. Chao and W. Chu, Vol. 7" (World Scientific, 2012) 115.

[3] European XFEL: URL available; http://www.xfel.eu

[4] G. Ciovati: Proc. IPAC2013, THYB201, Shanghai (2013), URL available; http://www.jacow.org.

[5] Y. Iwashita et al.: Proc. of LINAC2012 (2012) 156, URL available; http://www.jacow.org.

[6] D. Resche et al.: Proc. SRF conference, TUPO015 (2011), URL available; http://www.jacow.org.

[7] N. Walker et al.: Proc. SRF 2015, Whistler (2015), URL available; http://www.jacow.org. 
[8] O. Napoli, TTC meeting, CEA-Saclay, (2016), online available: https: //indico.in2p3.fr/event/12928/session/5/material/0/0.pdf

[9] FLASH: http://www.xfel.eu/overview/flash/

[10] J. Carwardine, et al., Workshop on Linac Operation with Long Bunch Train at DESY (2011), http://indico.desy.de/conferenceDisplay.py?confld=3007

[11] E. Harms, "CM2 recent results", TTC2014, KEK (2014), online available: https://indico.desy.de/sessionDisplay.py?sessionId=9\&confId=10663\#20141202

[12] S1-Global Report: KEK Report 2013-3 (2013).

[13] Y. Yamamoto et al., presented in this conference, ICHEP-2016.

[14] ATF: Accelerator Test Facility: http://atf.kek.jp

[15] Y. Honda, et.al., "Achievement of ultra-low emittance beam in the ATF damping ring", Phys. Rev. Lett. 92, 054802 (2004).

[16] P. Bambarde et al., Phys. Rev. STAB, 13, 0428001 (2010).

[17] G. White et al., Phys. Rev. Lett. 112, 034802 (2014).

[18] K. Kubo et al., "Towards ILC: Experiments at ATF", IPAC2015, Dresden, (2015), online available: http://accelconf.web.cern.ch/AccelConf/IPAC2014/papers/weza01.pdf

[19] T. Okugi, et al., IPAC2016, online available; http://accelconf.web.cern.ch/AccelConf/ipac2016/papers/thpmb043.pdf

[20] T. Shintake, Nucl. Instrum. Meth. A 311 (1992) 453-364.

[21] A. Grassellino, Linear Collider Workshop, 2016, Morioka, URL available: https://agenda.linearcollider.org/event/7371/sessions/4349/\#20161207

[22] A. Yamamoto and L. Evans, editor, "ILC Progress Report”, KEK-2015-004. (2015). 